\title{
COMMENTARY
}

\section{Fitting a square peg into a round hole: are the current Surviving Sepsis Campaign guidelines feasible for Africa?}

\author{
Shevin T Jacob*1, T Eoin West ${ }^{2}$ and Patrick Banura ${ }^{3}$ \\ See related research by Baelani et al., http://ccforum.com/content/15/1/R10
}

\begin{abstract}
In their article, Baelani and colleagues surveyed anesthesia providers from African low- and middleincome countries (LMICS) to evaluate whether or not the current Surviving Sepsis Campaign (SSC) guidelines are feasible in such resource-constrained settings. The authors report that an alarmingly low percentage of hospitals have the capacity to implement the SSC guidelines in their entirety but a higher percentage are able to implement the majority of SSC guidelines and grade 1 recommendations. In reality, the probability of adherence to SSC guidelines for septic management is even lower than reported, given that the majority of sepsis management in African LMICs is likely performed by non-intensivists outside of intensive care units. Efforts to address the challenges of managing severely ill patients in LMICs have recently been taken on by the World Health Organization. After reviewing available evidence for sepsis management predominantly from high-income countries, a panel of experts developed a consensus-based strategy tailored for resourcelimited settings. However, more research that can evaluate the challenges specific to sepsis management in LMICs and not currently addressed by the SSC guidelines is needed. Comprehensive, evidence-based guidelines combined with innovative approaches to sepsis management in LMICs are required to make a meaningful impact on worldwide sepsis survival.
\end{abstract}

In their article in this issue of Critical Care, Baelani and colleagues [1] attempt to determine whether or not

*Correspondence: sjacob2@uw.edu

'Division of Allergy and Infectious Diseases, Department of Medicine, University of Washington, Box 359927, 325 Ninth Avenue, Seattle, WA 98104, USA

Full list of author information is available at the end of the article
Surviving Sepsis Campaign (SSC) guidelines are implementable in African low- and middle-income countries (LMICs), where hospitals often lack the resources necessary for managing critically ill patients. When the SSC was introduced at the 2002 European Society of Intensive Care Medicine meeting in Barcelona, its main objective was to address the high worldwide mortality from sepsis by compiling a set of guidelines to standardize the management of this condition [2]. While adherence to SSC guidelines has since been associated with improved outcomes in high-income countries (HICs), where adequate resources are readily available [3], the authors highlight the fact that evaluation of the SSC guidelines' feasibility has never been conducted in LMICs, despite a high burden of infection and, consequently, sepsis [4]. Therefore, the authors conducted a survey of anesthesia providers attending the 2009 All Africa Anesthesia Congress, which compared the availability of resources necessary for implementation of SSC guidelines between $\mathrm{HICs}$ and African LMICs. The authors describe 307 attendants' responses from 185 hospitals in 24 African countries - low- (LIC) or middle(MIC) income countries - and 14 HICs.

Not surprisingly, the authors report a stark contrast in resources available for sepsis management between African countries and HICs and, to a lesser extent, between LICs and MICs within Africa. These differences occur with respect to the drugs, equipment, and disposable material required to implement SSC guidelines and correspond with an alarmingly low percentage of African hospitals (1.4\%) equipped to implement the entirety of SSC guidelines when compared with hospitals in HICs $(81.0 \%)$. More promising, however, is that the African hospitals could implement $67 \%$ of SSC guidelines and $75 \%$ of grade 1 recommendations.

In reality, this gap in resources is likely to be much wider than described. The survey, which targets anesthesia providers predominantly from university teaching or private hospitals, is biased toward a subset of providers 
who are more likely to have the fundamental resources required for sepsis management in comparison with providers who do not work within an intensive care unit (ICU). For example, whereas Baelani and colleagues reported that $93.8 \%$ of African hospitals represented in their survey always had oxygen available, another recent survey on oxygen delivery of 231 health centers and hospitals from 12 African countries reported that only $44 \%$ of facilities had uninterrupted access to an oxygen source [5]. Also, non-intensivists are more likely to manage septic patients in public hospitals from African LMICs. In our experience enrolling over 800 patients with severe sepsis in a national and regional referral hospital in Uganda, no patients were ever admitted or transferred to an ICU, despite the presence of an eightbed ICU at the national referral hospital $[6,7]$.

In early 2009, the World Health Organization convened a working group of external experts focused on tailoring sepsis management to address the challenges relevant to LMICs. The group has drawn on participant expertise in LMICs and available evidence from the HIC literature to create algorithms that focus primarily on hypotension (as an indicator of septic shock) and acute respiratory distress (as an indicator of acute lung injury). Although these algorithms represent the best effort to date, they lack data from research studies conducted in LMICs addressing the various issues specific to these settings. Nonetheless, the algorithms provide consensus-based recommendations in the absence of this evidence, particularly for addressing fundamental management questions such as the extent of aggressive fluid resuscitation when there is no recourse to mechanical ventilation and the choice of appropriate anti-microbial sepsis therapy in light of the microbial ecology of African LMICs [8]. A version of these guidelines was adapted for management of severely ill patients during the 2009 H1N1 pandemic and released for use to help countries prepare district hospital clinical teams [9]; the guidelines are supported by a training program that was field-tested in four countries in sub-Saharan Africa.

An additional consideration in the development of any guidelines should be the severe shortage of health workers in African LMICs, a circumstance that, at present, makes it impossible to provide the level of monitoring required for septic patients [10]. Ultimately, to make a meaningful impact on sepsis outcomes in African LMICs, increased availability of material resources for sepsis management will need to occur alongside health-worker training that focuses on the early identification, triage, monitoring, and treatment of severely ill, hospitalized patients.

Baelani and colleagues' appraisal of resources for sepsis management in LMICs provides a foundation on which future global efforts to develop feasible and cost-effective strategies that improve sepsis management can be customized for LMICs. With the underpinnings of the SSC and similar guidelines from HICs, it is time to develop comprehensive, evidence-based, and innovative strategies to overcome the barriers to improving sepsis survival in LMICs.

\section{Abbreviations}

HIC, high-income country; ICU, intensive care unit; LIC, low-income country; LMIC, low- and middle-income country; MIC, middle-income country; SSC, Surviving Sepsis Campaign.

\section{Competing interests}

The authors declare that they have no competing interests.

\section{Authors' information}

STJ, TEW, and PB are consultants for a sepsis working group convened in April 2009 by the Department of Global Alert and Response at the World Health Organization (WHO). Initially, the purpose of the group was to provide technical guidance for the management of severe sepsis syndromes to health providers from LMICs through the development of consensusbased guidelines. Since its inception, the group has broadened its scope to incorporate the management of all critical illness in resource-constrained settings and, as such, has dovetailed with the scope of work under other WHO groups, including the IMAI (Integrated Management of Adolescent and Adult IIIness) team and the Global Influenza Programme. During the emergence of pandemic influenza H1N1, the group developed emergency guidelines for the management of patients with severe respiratory distress and shock in district hospitals in limited-resource settings [9]. Since then, members of the group have been developing a protocol to evaluate the efficacy and effectiveness of these guidelines alongside a broader training curriculum developed by the IMAI team.

\section{Acknowledgments}

The authors would like to thank Matthew Lim and Sandy Gove for their input during the writing of this commentary.

\section{Author details}

'Division of Allergy and Infectious Diseases, Department of Medicine, University of Washington, Box 359927, 325 Ninth Avenue, Seattle, WA 98104, USA; ${ }^{2}$ Division of Pulmonary and Critical Care Medicine, Department of Medicine, University of Washington, Box 359640, 325 Ninth Avenue, Seattle, WA 98104, USA; ${ }^{M}$ Masaka Regional Referral Hospital, PO Box 18, Masaka, Uganda.

Published: 1 February 2011

\section{References}

1. Baelani I, Jochberger S, Laimer T, Otieno D, Kabutu J, Wilson I, Baker T, Dünser MW: Availability of critical care resources to treat patients with severe sepsis or septic shock in Africa: a self-reported, continent-wide survey of anaesthesia providers. Crit Care 2011, 15:R10.

2. Dellinger RP, Carlet JM, Masur H, Gerlach H, Calandra T, Cohen J, Gea-Banacloche J, Keh D, Marshall J, Parker MM, Ramsay G, Zimmerman JL, Vincent JL, Levy MM: Surviving Sepsis Campaign guidelines for management of severe sepsis and septic shock. Crit Care Med 2004 , 32:858-873.

3. Levy, MM, Dellinger RP, Townsend SR, Linde-Zwirble WT, Marshall JC, Bion J, Schorr C, Artigas A, Ramsay G, Beale R, Parker MM, Gerlach H, Reinhart K, Silva E, Harvey M, Regan S, Angus DC: The Surviving Sepsis Campaign: results of an international guideline-based performance improvement program targeting severe sepsis. Crit Care Med 2010, 38:367-374.

4. World Health Organization: The global burden of disease: 2004 update [http://www.who.int/healthinfo/global_burden_disease/2004_report_ update/en/index.html].

5. Belle J, Cohen H, Shindo N, Lim M, Velazquez-Berumen A, Ndihokubwayo J, Cherian M: Influenza preparedness in low-resource setting: a look at oxygen delivery in 12 African countries. J Infect Dev Ctries 2010, 4:419-424.

6. Jacob ST, Moore CC, Banura P, Pinkerton R, Meya D, Opendi P, Reynolds SJ, Kenya-Mugisha N, Mayanja-Kizza H, Scheld WM: Severe sepsis in two 
Ugandan hospitals: a prospective observational study of management and outcomes in a predominantly HIV-1 infected population. PLOS ONE 2009, 4:e7782

7. Jacob ST, Banura P, Baeten JM, Babigumira J, Moore CC, Meya D, Reynolds SJ, Wald A, Mayanja-Kizza H, Scheld WM: Enhanced fluid resuscitation improves survival in hospitalized Ugandan patients with severe sepsis. Paper presented at: 48th Annual Meeting of the Infectious Disease Society of America; 21-24 October 2010; Vancouver, Canada. Abstract 205.

8. Reddy EA, Shaw AV, Crump JA: Community-acquired bloodstream infections in Africa: a systematic review and meta-analysis. Lancet Infect Dis 2010, 10:417-432.

9. World Health Organization: Clinical management of adult patients with complications of pandemic influenza A (H1N1) 2009: emergency guidelines for the management of patients with severe respiratory distress and shock in district hospitals in limited-resource settings [http://whqlibdoc.who.int/publications/2010/9789241599610_eng.pdf].

10. World Health Organization: The world health report 2006: working together for health [http://www.who.int/whr/2006].

doi:10.1186/cc9981

Cite this article as: Jacob ST, et al.: Fitting a square peg into a round hole: are the current Surviving Sepsis Campaign guidelines feasible for Africa? Critical Care 2011, 15:117. 